\title{
Relationship between Social Support, Cultural Values, Family-Friendly Organizations and Psychological Well-Being among Turkish and the Dutch Nurses: The Role of Recovery
}

\author{
Hatem Öcel Collen \\ Fred Zijlstra \\ Faculty of Psychology \\ University of Maastricht \\ The Netherlands
}

\begin{abstract}
Purpose: The purpose of the study is to investigate the role of recovery after work in moderating the relationships between social support, cultural values, organizational characteristics, and psychological well-being. Design: Moderated regression analysis was used to analyze data. Methods: Cross-sectional self-reported questionnaire data was collected from Turkish and Dutch nurses (Turkish $N=333$, Dutch $N=323$ ). Social support, cultural values, family-friendly organizations, recovery strategies and well-being scales were used. Findings: We tested our hypotheses with the t test and multiple regression approach. The t test results showed the mean score of psychological well-being of Dutch participants $(t=3.07 ; p<.002)$; and that the mean score on recovery were significantly higher than those of Turkish participants $(t=3.90 ; p<.001)$. Results also showed that social support had an interaction effect with psychological detachment and relaxation on psychological well-being in the Turkish nurses; whereas in the Dutch nurses only social support and mastery experiences interacted on psychological well-being. Practical Implications: On the basis of our findings, it is advisable to focus interventions on enhancing using recovery strategies and well-being. Specific interventions should be directed at training in relaxation detachment from the work in free time.
\end{abstract}

Keywords: Social support, Cultural values, Family-friendly organizations and Psychological well-being

\section{Introduction}

Psychological well-being is very crucial in building prosperous societies. Focusing on psychological well-being at workplaces helps employees feel content, capable and satisfied with the job they do and provides an important advantage to societies. In general, psychological well-being - a very important component of wellness - is closely related to both the private life and professional life of individuals. When individuals maintain psychological well-being, they can cope with many challenges in their professional business life and private life more easily; while those who lack psychological well-being may have significant problems both in their business life and family life.

In this study, it is aimed to demonstrate which factors affect the psychological well-being of Dutch and Turkish nurses and whether workplace well-being strategies play a moderator role in maintaining psychological well-being. Nursing is a very demanding, tiring and stressful job that includes day and night shifts, in terms of the workload. Studies carried out in this field report that the psychological well-being of individuals in this occupational group is under risk. Although existing studies indicate that nursing is a quite stressful job, there are important cross national differences in terms of job demands and control over these demands, as well as in reported job stress (Schaufeli and Janczur, 1994). Turkish and Dutch nurses work in very different contexts. The Turkish health care context is characterized by one of the lowest nurse per capita ratios in Europe: 2 nurses per 1000 inhabitants. In comparison, the Dutch healthcare system has one of the highest ratios: 13 nurses per 1000 inhabitants (European Observatory on Health Care Systems, 2011). As a result, it is very important that nurses working under intensive stressful conditions maintain their psychological wellbeing and the factors that facilitate this are determined.

\section{Psychological Well-Being}

To Diener (2000), well-being is subjective and corresponds to happiness, in general. This subjective well-being comes out a result of one's evaluation of one's own life. Diener states that an individual who is subjectively good has a high level of life satisfaction and a minimum level of unpleasant emotions. However, an individual who is not subjectively good is not satisfied with his life and generally feels anxious and angry.

Diener (2000) points out that when a person is subject to positive or negative situations he/she detaches from normal emotional states for a while (feeling better or worse), but gets back to his/her normal state after some time. In other words, normal emotional state is the state when there is no excessive happiness or sorrow in one's life; unlike 
extraordinary emotional states when a person is very happy or very sad. Thus, it is the emotional state felt in normal circumstances that determine one's psychological well-being. Subjective well-being has two dimensions: being emotional and cognitive. Cognitive dimension accounts for the evaluation of one's life satisfaction in cognitive terms while emotional dimension relates to the maximum positive emotional state and minimum negative emotional state (Diener, Oishi, and Lucas, 2003).

Hypothesis 1: Turkish nurses' psychological well-being will be lower than Dutch.

\section{Recovery after Work as a Mediator}

It is an obvious fact that people need to have a rest to be able to get back to their previous state after a certain time spent at work. During a workday, employees confront several demands, physical, cognitive and emotional. In order to meet these demands, physical work, attention and concentration; physical and mental energy are necessary. When a work-day comes to an end, the person feels tired as he/she already consumes his/her existing physical and emotional sources (Meijman, Mulder and van Dormolen, 1992). This sense of tiredness brings out the need to have a rest so that the person can put into place the sources he/she consumes and can recover. The replacement of these resources is called recovery. Recovery after work is important because it helps people become ready to cope with new challenges and prevents this tiredness to accumulate and lead to permanent health problems in the end (Zijlstra and Sonnentag, 2006). This is exactly what we do when we have a rest or sleep after work: recovery. In a sense, it means to "recharge a battery".

According Zijlstra and Sonnentag (2006), it is necessary that working hours and breaks (resting time) follow one another so that work-rest cycle is maintained. However, there may not always be an opportunity to have a rest, therefore, it is important to seek other ways for recovery. One of these is to change the activity that is regularly done change of activity results in a relaxing effect on individuals. This is because the change of activity (such as changing physical activities with mental activities) means the change of demands. Physical activities require muscle workout; if they are replaced with mental activities, muscles recover and brainpower begins to take action.

Based on the literature on recovery, Sonnentag and Fritz (2007) introduce 4 different recovery strategies which are detaching psychologically from work, mastery, relaxation and control over leisure times. Although not being at work physically is important for recovery, it is not enough. What is meant by 'Detaching from work' is an individual's completely switching off work - not receiving phone calls or other duties about his/her job etc. Another strategy for recovery is relaxation that is about leisure activities. Relaxation is the state that comes as a result of activities (such as listening to music, walking in the nature etc.) chosen consciously to rest one's body and mind. The strategy of mastery refers to non-work activities which allow total distraction from the job as well as facilitating learning opportunities. Such non-work mastery activities increase individuals' positive affectivity. The last strategy handled in relation to recovery is control over leisure times. Within the scope of recovery, control is handled as the degree to which an individual decides the activities he will do in his spare time and how he will do them (Sonnentag and Fritz, 2007). Having control over spare time will improve self-efficacy and ensure the choice of activities that are liked or that facilitate recovery after work.

Recovery and psychological well-being are very closely-related concepts. Achieving recovery after work brings along psychological well-being in many cases. Literature shows that studies focusing on psychological well-being and work and rest activities have increased (Oerlemans, Bakker and Demerouti, 2014; Sonnentag and Zijlstra, 2006;Zijlstra and Sonnentag, 2006). Previous researches showed that some factors can facilitate recovery after work such as weekend and summer vacations (Cropley, and Zijlstra, 2011; Zijlstra, Cropley, and Rydstedt, 2014). Therefore, we assume that some other factors like social support, cultural differences and organizational characteristics also contribute to an individual's recovery process.

Hypothesis 2: Turkish nurses' levels of using recovery strategies will be lower than Dutch.

\section{Predictors of Recovery After Work}

\section{Social Support}

Social support provided by family members or friends contribute to recovery after-work. After-work recovery takes place when people spend time with other people or their best friends - for example when they go out for dinner, go to a party or chat with them on the phone. During such kind of social activities, social support channels of people remain open which contribute to their psychological well-being as well as recovery after-work (Sonnentag, 2001). In another study, similar results were obtained and it was reported that social activities (time spent with family and friends) provided social support to people and facilitated recovery after work. According to the findings of the same study, social support is an important source in decreasing the negative effects of job-related demands on psychological wellbeing (Bakker, Demerouti, and Euwema, 2005). 
Hypothesis 3: Social support will be positively related to the psychological well-being of Turkish and Dutch nurses and recovery strategies will moderate this relationship.

\section{Cultural Values}

The effects of cultural structure on the attitude and behavior of people is one of the subjects that have been investigated a several researchers (Hofstede, 1980; House et al. 1999-GLOBE). Lu, Gilmour and Kao (2001), state that cultural values are important components in determining the concept of well-being. In the study carried out by Gelfand et al. that covers 33 countries, cultures were categorized as tight and loose cultures, in terms of acting according to social norms. According to the findings of this study, Turkey is in Southern Asia group in terms acting according to social norms and is a country with tight cultural characteristics. Whereas the Netherlands shows medium level of cultural tightness together with Northern European countries (Gelfand, Raver, Nishii, Leslie, Lun, Lim, et al,2011). It is considered that this difference in cultural characteristics will show itself in recovery after work experiences and psychological well-beings of societies. To Kitayama and Markus (2000), psychological well-being is regarded to be more personal in societies with less tight characteristics in terms of acting according to social norms which relates to self-respect and personal happiness of individuals. In the societies that are stricter about acting according to social norms, psychological well-being is more relational, interpersonal and collective. In the societies that are deeply loyal to social norms (second group), individuals reciprocally follow these norms more (Kitayama and Markus, 2000). This reciprocal loyalty may sometimes threaten individuals' psychological well-being but sometimes help them cope with stressful conditions more easily by increasing the social support they feel in stressful conditions. It is thought that the cultural differences in business lives and family relations have effects on the recovery after work and psychological well-being of Dutch and Turkish nurses.

Hypothesis 4: Cultural values will be related to the psychological well-being of Turkish and Dutch nurses and recovery strategies will moderate this relationship.

\section{Organizational Characteristics}

When employee attitude and behavior - one of the fields of study in organizational psychology - are investigated, it is seen that one of the variables that are frequently emphasized is organizational characteristics. Some of these characteristics are the size of the organization, organizational policies, training program, study plans, conventional limits, organizational culture, family-friendly policies etc. Within the scope of the present study, family-friendly organizations that are considered to facilitate recovery after work and contribute to the psychological well-being of nurses will be encompassed as another organizational characteristic.

\section{Family-friendly Organizations}

In the changing conditions of the business world, maintaining job-related demands and family life together has caused a bigger stress on employees. In this regard, organizations have begun to develop some practices to reduce the pressure of work-family conflict on employees. For example, there are now kindergartens and nursing centers for the elderly in workplaces; the opportunity to take maternity leave; new regulations about more flexible working hours and financial support to families with children. The organizations having such practices are called family-friendly organizations. These organizations help their employees (especially female ones) manage the family-work relationship more easily. This is because women are responsible for the majority of housework and childcare, which leads to a conflict between their business lives and family lives (Greenberger, Goldberg, Hamill, O’Neil, and Payne, 1989). Studies indicate that the organizations that support family lives have positive effects on the psychological well-being of their employees (Kossek, Valcour, and Lirio, 2013; Thompson, and Prottas, 2005).

Hypothesis 5: Family-friendly organizations will be related to the psychological well-being of Turkish and Dutch nurses and recovery strategies will moderate this relationship.

Hypothesis 6: The moderating roles of recovery strategies will differentiate into a relationship between social support, family-friendly organizations, cultural values and psychological well-being among the Turkish and the Dutch samples.

The central aim of this study was to extend knowledge by examining the role of recovery after work in moderating the relationships between social support, cultural values, organizational characteristics, and psychological well-being. The present study compares psychological well-being in two relatively dissimilar countries in order to provide a better understanding of how we can improve psychological well-being in different settings. We assess the two countries' social support, cultural values, organizational characteristics to explain possible differences in psychological wellbeing.

\section{Method}

Sample 
This study examined the moderating role of recovery on the relationship between social support, cultural values, family-friendly organizations and well-being in Turkish $(\mathrm{N}=333)$ and Dutch $(\mathrm{N}=323)$ nurses. Participants' from Turkish sample ranged from 18 to 58 years in age (Mean=34.71 years $S D=8.42)$.

Participants from Turkish sample were 231 women and 102 men. Most of the participants (60.6\%) were married, 39.4 $\%$ were single. The age range of the Dutch participants was 17 - 65 and the mean age was32.48 (SD=11.01). Of 261 Dutch participants, 199 were female and 62 male. $66.9 \%$ of the Dutch participants were married, $26.9 \%$ were single.

\section{Measures}

Questionnaires were used for data collection. Well-being, cultural tightness looseness, recovery experiences, social support and family-friendly benefits measures were provided by the focal study participants. Items were in Turkish and Dutch languages respectively for Turkish and Dutch samples. The study was briefly explained to participants and they have been informed that involvement was completely voluntary and told them that they can withdraw at any time with no negative ramifications.

Wellbeing. Well-being was assessed using the scale developed by Diener et al. (2010) The Flourishing Scale. It is an 8-item self-report measure of flourishing (social-psychological prosperity). This scale is a new measure of well-being and it was called Psychological Wellbeing in an earlier publication, but the name was changed by Diener et al. (2010) because the scale includes content that goes beyond psychological well-being narrowly defined. Respondents are required to respond to each item (e.g., "I lead a purposeful and meaningful life") using a 7-point Likert scale (Strongly Disagree to Strongly Agree). Higher scores are representative of psychological well-being.

Cultural Tightness Looseness. Cultural tightness/looseness was measured with the 6 items questionnaire by using scale developed by Gelfand, et al. (2011). Each item (e.g., "There are many social norms that people are supposed to abide by in this country") was scored from strongly agree(6) to strongly disagree(1).

Recovery Experiences. Recovery activities were assessed with the scale developed by Sonnentag, Fritz (2007). It is a 16-item self-report measure of recovery after work. Items will be answered on a 5-point Likert scale $(1=$ Strongly disagree to 5=Strongly agree). Sample items were: "During evenings, I gain distance to my job requirements". Responses for each subscale were summed and averaged to get an average score for each sub-scale.

Social Support. The assessment of social support utilized a MSPSS developed by Zimet et al. (1988). The MSPSS is a 12-item instrument which measures perceived support from three sources such as family, friends and significant others. Items (e.g., "There is a special person who is around when I am in need") are scored on a 7-point rating scale ranging from very strongly disagree (1) to very strongly agree(7).

Family-friendly Benefits. Following Thompson \& Prottas (2005), family-friendly organizational characteristics is assessed by seven items (e.g., availability of a child-care or elder-care resource and referral service, financial assistance with child care, child-care center onsite or nearby, health insurance for family members); benefit availability was rated on a yes/no scale where $1=y e s$. Items will be summed to create a total score.

Control Variables. As control variables, we assessed age, gender, and marital status.

\section{Result}

Cronbach's alphas, descriptive statistics and Pearson's correlations were assessed (Table 1). T tests and regression analyses were done to compare Dutch and Turkish samples and test the relationship between social support, cultural values, family-friendly organisations, recovery and psychological well-being. The t test results showed that Dutch participants' mean score on of psychological well-being $(t=3.07 ; p<.002)$ and mean score on recovery were significantly higher than that of Turkish participants $(t=3.90 ; p<.001)$. These results indicate that as compared with Turkish nurses Dutch nurses have more well-being and recovery. There were significant differences between Turkish and Dutch participants' mean scores on the relaxation $(t=2.54 ; p<.01)$ and the mastery experiences $(t=7.86 ; p<.001)$ subscales of recovery. On the other hand, the differences between Turkish and Dutch participants' mean scores on the psychological detachment and the control during leisure time subscales of recovery were not significant.

Hypothesis 1 postulates that Turkish nurses' psychological well-being will be lower than Dutch. The findings supported this idea and Hypothesis 1 is accepted.

Hypothesis 2 postulates that Turkish nurses' levels of using recovery strategies will be lower than Dutch. According to results of the study, there are significant differences between Turkish and Dutch samples in terms of sub-dimensions of recovery scores. Thus, Hypothesis 2 also is accepted.

Furthermore, we evaluated the measurement of invariance across two countries. 
Table 1. Four models test invariances of measurement weights, structural co-variances, and measurement residuals

\begin{tabular}{|l|c|c|c|c|c|c|l|c|}
\hline Model & $\begin{array}{l}\text { NFI } \\
\text { Delta1 }\end{array}$ & $\begin{array}{l}\text { RFI } \\
\text { rho1 }\end{array}$ & $\begin{array}{l}\text { IFI } \\
\text { Delta2 }\end{array}$ & $\begin{array}{l}\text { TLI } \\
\text { rho2 }\end{array}$ & CFI & $\Delta$ CFI & RMSEA & AIC \\
\hline Unconstrained &, 668 &, 646 &, 766 &, 748 &, 764 & - &, 049 & 4357,475 \\
\hline Measurement weights &, 657 &, 643 &, 756 &, 744 &, 755 & 0,009 &, 050 & 4418,743 \\
\hline Structural covariance &, 649 &, 638 &, 748 &, 739 &, 747 & 0,008 &, 050 & 4416,435 \\
\hline Measurement residuals &, 614 &, 612 &, 710 &, 708 &, 710 & 0,04 &, 053 & 4482,226 \\
\hline
\end{tabular}

Browne and Cudeck (1993) suggested that values of the RMSEA of .05 or less indicate a close fit, and .08 or less indicate adequate fit. For the RMSEA, values less than 0.05 and 0.08 reflect a close fit and a reasonable fit to the data, respectively (Marsh et al., 2004). According to RMSEA values, first three CFA models indicate good fit to the data, whereas the model which tests invariances of measurements residuals has a reasonable fit to the data.

\section{Regression Analysis}

In this study, the moderator regression analyses were done to examine moderator role of recovery strategies psychological detachment, relaxation, mastery experiences and control- between social support, cultural values, familyfriendly organisations and well-being. Moderator regression analyses were conducted based on Aiken and West's (1991) advices.

Firstly, we controlled for the variables gender, age, and marital status because these variables were correlated with both predictors and outcomes.First of all the results revealed that, the interaction between social support and psychological detachment $(\beta=.88, \mathrm{t}=2.55, \mathrm{R} 2$ change $=.02$, Fchange $7,321=9.18, \mathrm{p}<.000)$ and relaxation $(\beta=1.43, \mathrm{t}=3.65, \mathrm{R} 2$ change $=.03$, Fchange $7,321=10.89, \mathrm{p}<.000)$ on psychological well-being were significant in Turkish sample. Secondly, results also showed that social support and mastery experiences interacted on psychological well-being in Dutch sample $(\beta=.66, \mathrm{t}=1.89, \mathrm{R} 2$ change $=.02$, Fchange $6,325=1.43, \mathrm{p}<.05)$. Table 4 shows the results.

Hypothesis 3 claims that social support will be positively related to the psychological well-being of Turkish and Dutch nurses and recovery strategies will moderate this relationship. Social support emerged as a strong predictor of psychological well-being in both Turkish and Dutch nurses (respectively $\beta=.39, t=7.06, p<.000 ; \beta=.11, t=2.12, p<$ .03). Based on the moderator regression analysis results, there is a significant interaction between social support and two sub-dimensions of recovery (psychological detachment and relaxation) among Turkish but non-significant in Dutch. Based on this result, Hypothesis 3 is partially supported. See Table 2 and 3 for the results.

Hypothesis 4 claims that cultural values will be related to the psychological well-being of Turkish and Dutch nurses and recovery strategies will moderate this relationship. Results showed that cultural values predict psychological wellbeing in Turkish sample $(\beta=.11, \mathrm{t}=2.14, \mathrm{p}<.03)$ but not in Dutch counterparts. Additionally, there is no significant interaction between cultural values and recovery strategies in both Turkish and Dutch sample. Therefore, Hypothesis 4 is partially supported. 
Table 2. Mean (M), Standard Deviation (SD), Internal Consistencies (Cronbach's $\alpha$ ) and Correlations

\begin{tabular}{|c|c|c|c|c|c|c|c|c|c|c|c|c|c|}
\hline Variables & $\mathrm{M}$ & SD & $\alpha$ & 1 & 2 & 3 & 4 & 5 & 6 & 7 & 8 & 9 & 10 \\
\hline 1.Age & $\begin{array}{l}\text { (T) } 34.71 \\
\text { (D) } 32.48\end{array}$ & $\begin{array}{l}8.42 \\
11.01\end{array}$ & & & & & & & & & & & \\
\hline 2.Gender & $\begin{array}{l}\text { (T) } 1.30 \\
\text { (D) } 1.19\end{array}$ & $\begin{array}{l}.46 \\
.39\end{array}$ & & $\begin{array}{l}-.03 \\
-.02\end{array}$ & & & & & & & & & \\
\hline 3.Marital Status & $\begin{array}{l}\text { (T) } 1.60 \\
\text { (D) } 1.77\end{array}$ & $\begin{array}{l}.48 \\
.53\end{array}$ & & $\begin{array}{l}.52^{* *} \\
.33^{* *}\end{array}$ & $\begin{array}{l}.03 \\
.03\end{array}$ & & & & & & & & \\
\hline 4.Social Support & $\begin{array}{l}\text { (T) } 49.43 \\
\text { (D) } 49.47\end{array}$ & $\begin{array}{l}8.22 \\
7.93\end{array}$ & $\begin{array}{l}.91 \\
92\end{array}$ & $\begin{array}{l}.06 \\
-.12 *\end{array}$ & $\begin{array}{l}-.04 \\
-.01\end{array}$ & $\begin{array}{l}.08 \\
.02\end{array}$ & & & & & & & \\
\hline $\begin{array}{l}\text { 5.Family-Friendly } \\
\text { Organizations }\end{array}$ & $\begin{array}{l}\text { (T) } 2.69 \\
\text { (D) } 2.52\end{array}$ & $\begin{array}{l}1.65 \\
1.69\end{array}$ & - & $\begin{array}{l}.02 \\
.16^{* *}\end{array}$ & $\begin{array}{l}.01 \\
.13^{*}\end{array}$ & $\begin{array}{l}.10 \\
-.24 * *\end{array}$ & $\begin{array}{l}.08 \\
.08\end{array}$ & & & & & & \\
\hline 6.Cultural Values & $\begin{array}{l}\text { (T) } 21.15 \\
\text { (D) } 24.70\end{array}$ & $\begin{array}{l}5.87 \\
4.65\end{array}$ & $\begin{array}{l}.74 \\
.66\end{array}$ & $\begin{array}{l}-.10 \\
.12 *\end{array}$ & $\begin{array}{l}.04 \\
-.03\end{array}$ & \begin{tabular}{l|}
-.08 \\
.04
\end{tabular} & $\begin{array}{l}.19^{* * *} \\
.03\end{array}$ & $\begin{array}{l}.05 \\
.06\end{array}$ & & & & & \\
\hline Recovery After Work & & & & & & & & & & & & & \\
\hline $\begin{array}{l}\text { 7.Psychological } \\
\text { Detachment }\end{array}$ & $\begin{array}{l}\text { (T) } 11.55 \\
\text { (D) } 11.48\end{array}$ & $\begin{array}{l}4.39 \\
3.27\end{array}$ & $\begin{array}{l}.88 \\
.78\end{array}$ & $\begin{array}{l}.20 * * \\
-.03\end{array}$ & $\begin{array}{l}.01 \\
-.01\end{array}$ & $\begin{array}{l}.12^{*} \\
.16^{* *}\end{array}$ & $\begin{array}{l}.04 \\
.07\end{array}$ & $\begin{array}{l}-.08 \\
.05\end{array}$ & $\begin{array}{l}-.04 \\
-.07\end{array}$ & & & & \\
\hline 8.Relaxation & $\begin{array}{l}\text { (T) } 13.03 \\
\text { (D) } 13.77\end{array}$ & $\begin{array}{l}4.11 \\
3.21 \\
\end{array}$ & $\begin{array}{l}.86 \\
.78\end{array}$ & $\begin{array}{l}-.08 \\
-.03 \\
\end{array}$ & $\begin{array}{l}.02 \\
.01\end{array}$ & $\begin{array}{l}.07 \\
.18 * *\end{array}$ & $\begin{array}{l}.19^{* *} \\
.17^{* *}\end{array}$ & $\begin{array}{l}.03 \\
.06\end{array}$ & $\begin{array}{l}.13^{*} \\
.03\end{array}$ & $\begin{array}{l}.42 * * \\
.51 * * \\
\end{array}$ & & & \\
\hline 9.Mastery Experiences & $\begin{array}{l}\text { (T) } 11.99 \\
\text { (D) } 13.95\end{array}$ & $\begin{array}{l}3.40 \\
2.96\end{array}$ & $\begin{array}{l}.79 \\
.78\end{array}$ & $\begin{array}{l}-.04 \\
-.01\end{array}$ & $\begin{array}{l}.03 \\
-.03\end{array}$ & $\begin{array}{l}-.07 \\
-.07\end{array}$ & $\begin{array}{l}.17^{* *} \\
.19^{* *}\end{array}$ & $\begin{array}{l}.07 \\
.13^{*}\end{array}$ & $\begin{array}{l}.06 \\
.01\end{array}$ & $\begin{array}{l}.00 \\
.08\end{array}$ & $\begin{array}{l}.35 * * \\
.21 * *\end{array}$ & & \\
\hline 10.Control & $\begin{array}{l}\text { (T) } 14.54 \\
\text { (D) } 14.86\end{array}$ & $\begin{array}{l}3.33 \\
2.73\end{array}$ & $\begin{array}{l}.82 \\
.71\end{array}$ & $\begin{array}{l}-.04 \\
-.07\end{array}$ & $\begin{array}{l}-.04 \\
.01\end{array}$ & $\begin{array}{l}.03 \\
-.16^{* *}\end{array}$ & $\begin{array}{l}.23^{* *} \\
.23^{* *}\end{array}$ & $\begin{array}{l}.10^{*} \\
.20 * *\end{array}$ & $\begin{array}{l}.13^{*} \\
.07\end{array}$ & $\begin{array}{l}.21 * * \\
.38 * *\end{array}$ & $\begin{array}{l}.40^{* * *} \\
.61 * *\end{array}$ & $\begin{array}{l}.39 * * \\
.26 * *\end{array}$ & \\
\hline 11. Well-Being & $\begin{array}{l}\text { (T) } 43.90 \\
\text { (D) } 45.54\end{array}$ & $\begin{array}{l}7.01 \\
6.65\end{array}$ & $\begin{array}{l}.83 \\
.86\end{array}$ & $\begin{array}{l}.02 \\
-.05\end{array}$ & $\begin{array}{l}-.00 \\
-.02\end{array}$ & $\begin{array}{l}.05 \\
-.03\end{array}$ & $\begin{array}{l}.38^{* *} \\
.50^{* *}\end{array}$ & $\begin{array}{l}.15^{* *} \\
.05\end{array}$ & $\begin{array}{l}.17^{* *} \\
.05\end{array}$ & $\begin{array}{l}.00 \\
.17 * *\end{array}$ & $\begin{array}{l}.16^{* * *} \\
.24^{* *}\end{array}$ & $\begin{array}{l}.32^{* *} \\
.27^{* *}\end{array}$ & $\begin{array}{l}.32 * * \\
34 * *\end{array}$ \\
\hline
\end{tabular}

$* \mathrm{~T}=$ Turkish; $\mathrm{D}=\mathrm{Dutch} ;{ }^{*} p<.05, * * p<.01$

Hypothesis 5 states that family-friendly organizations will be related to the psychological well-being of Turkish and Dutch nurses and recovery strategies will moderate this relationship. Similarly results showed that family-friendly organizations predict psychological well-being only in Turkish sample $(\beta=.11, \mathrm{t}=2.30, \mathrm{p}<.02)$. There is no significant interaction between family-friendly organizations and recovery strategies in both Turkish and Dutch sample (see Table 3, 4, 5 and 6). With this regard, Hypothesis 5 is also partially supported.

Hypothesis 6 states that the moderating roles of recovery strategies will differentiate into a relationship between social support, family-friendly organizations, cultural values and psychological well-being among the Turkish and the Dutch samples. Research results showed that social support had an interaction effect with psychological detachment and relaxation on psychological well-being in the Turkish nurses (see in Table 3 and 4), whereas in the Dutch nurses only social support and mastery experiences interacted on psychological well-being(see in Table 5). On the other hand, there was no moderator role of recovery strategies on relationship between family-friendly organizations, cultural values and psychological well-being. Consequently results revealed that moderating roles of recovery strategies differentiate into a relationship between social support, family-friendly organizations and cultural values among the Turkish and the Dutch samples. Based on these results Hypothesis 6 is supported. 
Table 3. Moderated Regression Analysis Results for Psychological Well-Being (Social Support, Family-Friendly Organizations, Cultural Values and Psychological Detachment)

\begin{tabular}{|c|c|c|c|c|c|c|c|c|}
\hline Variables & & $\bar{B}$ & $\overline{\bar{\beta}}$ & $\overline{T T}$ & $P$ & $R^{2}$ & $R_{C H A N}$ & $\overline{F F}$ \\
\hline $\begin{array}{l}\text { Step } 1 . \\
\text { Age } \\
\text { Gender } \\
\text { Marital Status }\end{array}$ & $\begin{array}{l}T \\
D \\
T \\
D \\
T \\
D \\
T \\
D\end{array}$ & $\begin{array}{l}-.005 \\
-.10 \\
.071 \\
-.90 \\
.733 \\
.31 \\
\end{array}$ & \begin{tabular}{|l|}
-.006 \\
-.08 \\
.005 \\
-.02 \\
.05 \\
-.01 \\
\end{tabular} & \begin{tabular}{|l}
-.09 \\
-1.35 \\
.08 \\
-.44 \\
.77 \\
.19 \\
\end{tabular} & $\begin{array}{l}.92 \\
.17 \\
.93 \\
.65 \\
.43 \\
.84\end{array}$ & $\begin{array}{l}.009 \\
.006\end{array}$ & $\begin{array}{l}.009 \\
.006\end{array}$ & \begin{tabular}{|l|}
.74 \\
.68
\end{tabular} \\
\hline $\begin{array}{l}\text { Step } 2 . \\
\text { Social Support } \\
\text { Psychological Detachment }\end{array}$ & $\begin{array}{l}T \\
D \\
T \\
D \\
T \\
D\end{array}$ & $\begin{array}{l}.30 \\
.13 \\
-1.25 \\
.11 \\
\end{array}$ & $\begin{array}{l}.38 \\
.11 \\
-.78 \\
.02 \\
\end{array}$ & \begin{tabular}{|l|}
7.29 \\
2.04 \\
-2.55 \\
.46 \\
\end{tabular} & $\begin{array}{l}.000 \\
.04 \\
.01 \\
.64 \\
\end{array}$ & $\begin{array}{l}.15 \\
.02\end{array}$ & $\begin{array}{l}.15 \\
.02\end{array}$ & \begin{tabular}{|l|}
9.45 \\
1.36
\end{tabular} \\
\hline $\begin{array}{l}\text { Step } 3 . \\
\text { Soci Sup X Pay. Detach. }\end{array}$ & $\begin{array}{l}\mathrm{T} \\
\mathrm{D} \\
\mathrm{T} \\
\mathrm{D}\end{array}$ & $\begin{array}{l}.02 \\
-.007\end{array}$ & \begin{tabular}{|l}
.88 \\
-.10
\end{tabular} & \begin{tabular}{|l|}
2.55 \\
-.33
\end{tabular} & $\begin{array}{l}.01 \\
.74\end{array}$ & $\begin{array}{l}.17 \\
.02\end{array}$ & \begin{tabular}{|l|}
.02 \\
.00
\end{tabular} & \begin{tabular}{|l|}
9.18 \\
1.15
\end{tabular} \\
\hline Variables & & $B$ & $\beta$ & $t$ & $p$ & $R^{2}$ & $R_{\text {eher }}^{2}$ & $F$ \\
\hline $\begin{array}{l}\text { Step } 1 . \\
\text { Age } \\
\text { Gender } \\
\text { Marital Status }\end{array}$ & $\begin{array}{l}T \\
D \\
T \\
D \\
T \\
D \\
T \\
D\end{array}$ & $\begin{array}{l}-.005 \\
-.10 \\
.071 \\
-.90 \\
.733 \\
.31\end{array}$ & $\begin{array}{l}-.006 \\
-.08 \\
.005 \\
-.02 \\
.05 \\
.01 \\
\end{array}$ & \begin{tabular}{|l}
-.09 \\
-1.35 \\
.08 \\
-.44 \\
.77 \\
.19 \\
\end{tabular} & $\begin{array}{l}.92 \\
.17 \\
.93 \\
.65 \\
.43 \\
.84\end{array}$ & \begin{tabular}{|l}
.009 \\
.006
\end{tabular} & $\begin{array}{l}.009 \\
.006\end{array}$ & \begin{tabular}{|l}
.74 \\
.68
\end{tabular} \\
\hline $\begin{array}{l}\text { Step } 2 . \\
\text { Family-Friendly Organizations } \\
\text { Psychological Detachment }\end{array}$ & $\begin{array}{l}T \\
D \\
T \\
D \\
T \\
D\end{array}$ & $\begin{array}{l}.59 \\
.26 \\
-.17 \\
.18 \\
\end{array}$ & $\begin{array}{l}.14 \\
.03 \\
-.11 \\
.04\end{array}$ & \begin{tabular}{|l|}
2.52 \\
.53 \\
-1.09 \\
.73 \\
\end{tabular} & $\begin{array}{l}.01 \\
.59 \\
.27 \\
.46\end{array}$ & \begin{tabular}{|l}
.02 \\
.009
\end{tabular} & $\begin{array}{l}.02 \\
.01\end{array}$ & $\begin{array}{l}1.56 \\
.58\end{array}$ \\
\hline $\begin{array}{l}\text { Step } 3 . \\
\text { Fam Frien, Oxgan X.Pow, Detach. }\end{array}$ & $\begin{array}{l}\text { T } \\
\text { D } \\
\text { T } \\
\text { D }\end{array}$ & $\begin{array}{l}.07 \\
.13\end{array}$ & $\begin{array}{l}.24 \\
.19\end{array}$ & \begin{tabular}{|l}
1.48 \\
.96
\end{tabular} & $\begin{array}{l}.13 \\
.33\end{array}$ & $\begin{array}{l}.03 \\
.01\end{array}$ & $\begin{array}{l}.01 \\
.01\end{array}$ & $\begin{array}{l}1.66 \\
.63\end{array}$ \\
\hline Variables & & $B$ & $\beta$ & $t$ & $p$ & $R^{2}$ & $R_{\text {eher. }}^{2}$ & $F$ \\
\hline $\begin{array}{l}\text { Step } 1 . \\
\text { Age } \\
\text { Gender } \\
\text { Marital Status }\end{array}$ & $\begin{array}{l}T \\
D \\
T \\
D \\
T \\
D \\
T \\
D\end{array}$ & $\begin{array}{l}-.005 \\
-.10 \\
.071 \\
-.90 \\
.733 \\
.31\end{array}$ & $\begin{array}{l}-.006 \\
-.08 \\
.005 \\
-.02 \\
.05 \\
.01 \\
\end{array}$ & $\begin{array}{l}-.09 \\
1.35 \\
.08 \\
-.44 \\
.77 \\
.19 \\
\end{array}$ & $\begin{array}{l}.92 \\
.17 \\
.93 \\
.65 \\
.43 \\
.84\end{array}$ & $\begin{array}{l}.009 \\
.006\end{array}$ & \begin{tabular}{|l}
.009 \\
.006
\end{tabular} & \begin{tabular}{|l|}
.74 \\
.68 \\
\end{tabular} \\
\hline $\begin{array}{l}\text { Step } 2 . \\
\text { Cultural Values } \\
\text { Psychological Detachment }\end{array}$ & $\begin{array}{l}T \\
D \\
T \\
D \\
T \\
D \\
\end{array}$ & $\begin{array}{l}.22 \\
.21 \\
-.07 \\
.20 \\
\end{array}$ & \begin{tabular}{|l}
.19 \\
.07 \\
-.04 \\
.04 \\
\end{tabular} & \begin{tabular}{|l|}
3.41 \\
1.25 \\
-.22 \\
.82 \\
\end{tabular} & $\begin{array}{l}.001 \\
.20 \\
.82 \\
.41 \\
\end{array}$ & \begin{tabular}{|l|}
.04 \\
.01
\end{tabular} & \begin{tabular}{|l|}
.04 \\
.001
\end{tabular} & $\begin{array}{l}2.46 \\
.84\end{array}$ \\
\hline $\begin{array}{l}\text { Step } 3 . \\
\text { Cult. JaluX X5x. Detach. }\end{array}$ & $\begin{array}{l}\mathrm{T} \\
\mathrm{D} \\
\mathrm{T} \\
\mathrm{D}\end{array}$ & $\begin{array}{l}.04 \\
-.007 \\
\end{array}$ & \begin{tabular}{|l}
.06 \\
-.04 \\
\end{tabular} & \begin{tabular}{|l}
.26 \\
-.12 \\
\end{tabular} & $\begin{array}{l}.79 \\
.89 \\
\end{array}$ & \begin{tabular}{|l|}
.04 \\
.01
\end{tabular} & $\begin{array}{l}.00 \\
.00\end{array}$ & $\begin{array}{l}2.11 \\
.70\end{array}$ \\
\hline
\end{tabular}


Table 4. Moderated Regression Analysis Results for Psychological Well-Being (Social Support, Family-Friendly Organizations, Cultural Values and Relaxation)

\begin{tabular}{|c|c|c|c|c|c|c|c|c|}
\hline Variables & & $\overline{\bar{B}}$ & $\overline{\beta \beta}$ & $\bar{T}$ & $\overline{P P}$ & $\overline{R^{2}}$ & $R_{C A N}$ CR: & $\bar{F}$ \\
\hline $\begin{array}{l}\text { Step } 1 . \\
\text { Age } \\
\text { Gender } \\
\text { Marital Status }\end{array}$ & $\begin{array}{l}T \\
D \\
T \\
D \\
T \\
D \\
T \\
D\end{array}$ & $\begin{array}{l}-.005 \\
-.10 \\
.071 \\
-.90 \\
.733 \\
.31\end{array}$ & $\begin{array}{l}-.006 \\
-.08 \\
.005 \\
-.02 \\
.05 \\
-.01\end{array}$ & $\begin{array}{l}-.09 \\
-1.35 \\
.08 \\
-.44 \\
.77 \\
.19\end{array}$ & $\begin{array}{l}.92 \\
.17 \\
.93 \\
.65 \\
.43 \\
.84\end{array}$ & $\begin{array}{l}.009 \\
.006\end{array}$ & $\begin{array}{l}.009 \\
.006\end{array}$ & $\begin{array}{l}.74 \\
.68\end{array}$ \\
\hline $\begin{array}{l}\text { Step } 2 . \\
\text { Social Support } \\
\text { Relaxcation }\end{array}$ & \begin{tabular}{l|}
$T$ \\
$D$ \\
$T$ \\
$D$ \\
$T$ \\
$D$ \\
\end{tabular} & \begin{tabular}{l|}
.29 \\
.13 \\
-1.77 \\
.16 \\
\end{tabular} & $\begin{array}{l}.36 \\
.11 \\
-1.74 \\
.03\end{array}$ & \begin{tabular}{|l|}
6.74 \\
1.96 \\
-3.29 \\
.63 \\
\end{tabular} & $\begin{array}{l}.000 \\
.05 \\
.001 \\
.52\end{array}$ & $\begin{array}{l}.16 \\
.02\end{array}$ & $\begin{array}{l}.16 \\
.02\end{array}$ & $\begin{array}{l}10.09 \\
1.40\end{array}$ \\
\hline $\begin{array}{l}\text { Step } 3 . \\
\text { Soci SupuX Relaxation }\end{array}$ & \begin{tabular}{l|}
$\mathrm{T}$ \\
$\mathrm{D}$ \\
$\mathrm{T}$ \\
$\mathrm{D}$
\end{tabular} & $\begin{array}{l}.03 \\
.02\end{array}$ & $\begin{array}{l}1.43 \\
.40\end{array}$ & $\begin{array}{l}3.65 \\
1.24\end{array}$ & $\begin{array}{l}.000 \\
.21\end{array}$ & $\begin{array}{l}.19 \\
.02\end{array}$ & $\begin{array}{l}.03 \\
.02\end{array}$ & $\begin{array}{l}10.89 \\
1.43\end{array}$ \\
\hline Variables & & $B$ & $B$ & $t$ & $p$ & $R^{2}$ & $R_{\text {eher. }}^{2}$ & $F$ \\
\hline $\begin{array}{l}\text { Step } 1 . \\
\text { Age } \\
\text { Gender } \\
\text { Marital Status }\end{array}$ & $\begin{array}{l}T \\
D \\
T \\
D \\
T \\
D \\
T \\
D\end{array}$ & $\begin{array}{l}-.005 \\
-.10 \\
.071 \\
-.90 \\
.733 \\
.31\end{array}$ & $\begin{array}{l}-.006 \\
-.08 \\
.005 \\
-.02 \\
.05 \\
-.01\end{array}$ & $\begin{array}{l}-.09 \\
-1.35 \\
.08 \\
-.44 \\
.77 \\
.19\end{array}$ & $\begin{array}{l}.92 \\
.17 \\
.93 \\
.65 \\
.43 \\
.84\end{array}$ & \begin{tabular}{|l|}
.009 \\
006
\end{tabular} & $\begin{array}{l}.009 \\
.006\end{array}$ & $\begin{array}{l}.74 \\
.68\end{array}$ \\
\hline $\begin{array}{l}\text { Step } 2 . \\
\text { Family-Friendly Org. } \\
\text { Relaxcation }\end{array}$ & \begin{tabular}{l|}
$T$ \\
$D$ \\
$T$ \\
$D$ \\
$T$ \\
$D$
\end{tabular} & \begin{tabular}{l|}
.56 \\
.26 \\
.002 \\
.26
\end{tabular} & $\begin{array}{l}.13 \\
.03 \\
.001 \\
.05\end{array}$ & $\begin{array}{l}2.41 \\
.52 \\
.01 \\
1.01\end{array}$ & $\begin{array}{l}.01 \\
.60 \\
.99 \\
.31\end{array}$ & $\begin{array}{l}.05 \\
.001\end{array}$ & $\begin{array}{l}.05 \\
.001\end{array}$ & $\begin{array}{l}3.20 \\
.67\end{array}$ \\
\hline $\begin{array}{l}\text { Step } 3 . \\
\text { Fam. Erien. Organ X.Relaxation }\end{array}$ & \begin{tabular}{l|}
$\mathrm{T}$ \\
$\mathrm{D}$ \\
$\mathrm{T}$ \\
$\mathrm{D}$
\end{tabular} & $\begin{array}{l}.11 \\
14\end{array}$ & $\begin{array}{l}.41 \\
26\end{array}$ & $\begin{array}{l}1.82 \\
1.02\end{array}$ & $\begin{array}{l}.99 \\
30\end{array}$ & \begin{tabular}{l|}
.06 \\
.001
\end{tabular} & $\begin{array}{l}.01 \\
.001\end{array}$ & $\begin{array}{l}3.24 \\
.74\end{array}$ \\
\hline Variables & & $B$ & $\beta$ & $t$ & $p$ & $R^{2}$ & $R_{\text {eher. }}^{2}$ & $F$ \\
\hline $\begin{array}{l}\text { Step } 1 . \\
\text { Age } \\
\text { Gender } \\
\text { Marital Status }\end{array}$ & $\begin{array}{l}T \\
D \\
T \\
D \\
T \\
D \\
T \\
D\end{array}$ & \begin{tabular}{l|}
-.005 \\
-.10 \\
.071 \\
-.90 \\
.733 \\
.31 \\
\end{tabular} & $\begin{array}{l}-.006 \\
-.08 \\
.005 \\
-.02 \\
.05 \\
-.01\end{array}$ & $\begin{array}{l}-.09 \\
-1.35 \\
.08 \\
-.44 \\
.77 \\
.19\end{array}$ & $\begin{array}{l}.92 \\
.17 \\
.93 \\
.65 \\
.43 \\
.84\end{array}$ & \begin{tabular}{|l|}
.009 \\
006
\end{tabular} & $\begin{array}{l}.009 \\
.006\end{array}$ & $\begin{array}{l}.74 \\
.68\end{array}$ \\
\hline $\begin{array}{l}\text { Step } 2 . \\
\text { Cultural Values } \\
\text { Relaxcation }\end{array}$ & \begin{tabular}{l|}
$T$ \\
$D$ \\
$T$ \\
$D$ \\
$T$ \\
$D$ \\
\end{tabular} & $\begin{array}{l}.20 \\
.20 \\
.26 \\
.25\end{array}$ & $\begin{array}{l}.17 \\
.06 \\
.15 \\
.05\end{array}$ & $\begin{array}{l}.3 .05 \\
1.16 \\
2.78 \\
.97\end{array}$ & $\begin{array}{l}.002 \\
.24 \\
.006 \\
.32\end{array}$ & \begin{tabular}{l|}
.05 \\
.001
\end{tabular} & $\begin{array}{l}.05 \\
.001\end{array}$ & $\begin{array}{l}3.81 \\
.89\end{array}$ \\
\hline $\begin{array}{l}\text { Step } 3 . \\
\text { Cult. Valu.X Relaxation }\end{array}$ & \begin{tabular}{|l|}
$\mathrm{T}$ \\
$\mathrm{D}$ \\
$\mathrm{T}$ \\
$\mathrm{D}$ \\
\end{tabular} & $\begin{array}{l}.01 \\
.04\end{array}$ & $\begin{array}{l}.34 \\
.34 \\
\end{array}$ & $\begin{array}{l}1.26 \\
.87 \\
\end{array}$ & $\begin{array}{l}.20 \\
.38 \\
\end{array}$ & $\begin{array}{l}.06 \\
.001\end{array}$ & $\begin{array}{l}.01 \\
.001\end{array}$ & $\begin{array}{l}3.50 \\
.87\end{array}$ \\
\hline
\end{tabular}

${ }^{*} \mathrm{~T}=$ Turkish, $\mathrm{D}=$ Dutch; ${ }^{*} \mathrm{p}<, 05 ;{ }^{* *} \mathrm{p}<, 01$; $^{* \star *} \mathrm{p}<000$ 
Table 5 Moderated Regression Analysis Results for Psychological Well-Being(Social Support, Family-Friendly Organizations, Cultural Values and Mastery Experiences)

\begin{tabular}{|c|c|c|c|c|c|c|c|c|}
\hline Variables & & $\overline{B B}$ & $\overline{\bar{\beta}}$ & 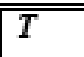 & $\overline{P P}$ & 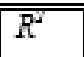 & 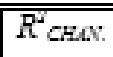 & 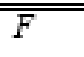 \\
\hline $\begin{array}{l}\text { Step } 1 . \\
\text { Age } \\
\text { Gender } \\
\text { Marital Status }\end{array}$ & $\begin{array}{l}T \\
D \\
T \\
D \\
T \\
D \\
T \\
D\end{array}$ & $\begin{array}{l}-.005 \\
-.10 \\
.071 \\
-.90 \\
.733 \\
.31\end{array}$ & $\begin{array}{l}-.006 \\
-.08 \\
.005 \\
-.02 \\
.05 \\
-.01\end{array}$ & $\begin{array}{l}-.09 \\
-1.35 \\
.08 \\
-.44 \\
.77 \\
.19\end{array}$ & $\begin{array}{l}.92 \\
.17 \\
.93 \\
.65 \\
.43 \\
.84\end{array}$ & $\begin{array}{l}.009 \\
.006\end{array}$ & \begin{tabular}{|l|}
.009 \\
.006
\end{tabular} & $\begin{array}{l}.74 \\
.68\end{array}$ \\
\hline $\begin{array}{l}\text { Step } 2 . \\
\text { Social Support } \\
\text { Mastery Experiences }\end{array}$ & $\begin{array}{l}T \\
D \\
T \\
D \\
T \\
D \\
\end{array}$ & $\begin{array}{l}.27 \\
.12 \\
.55 \\
.42 \\
\end{array}$ & $\begin{array}{l}.33 \\
.06 \\
.26 \\
.08 \\
\end{array}$ & $\begin{array}{l}6.61 \\
1.90 \\
5.27 \\
1.55 \\
\end{array}$ & $\begin{array}{l}.000 \\
.05 \\
.000 \\
.12 \\
\end{array}$ & $\begin{array}{l}.22 \\
.02\end{array}$ & $\begin{array}{l}.22 \\
.02\end{array}$ & $\begin{array}{l}14.92 \\
1.40\end{array}$ \\
\hline $\begin{array}{l}\text { Step } 3 . \\
\text { Soci Sup.X. Mastery Exper. }\end{array}$ & $\begin{array}{l}\mathrm{T} \\
\mathrm{D} \\
\mathrm{T} \\
\mathrm{D}\end{array}$ & $\begin{array}{l}.02 \\
-.04 \\
\end{array}$ & $\begin{array}{l}.72 \\
.66 \\
\end{array}$ & \begin{tabular}{|l|l}
1.70 \\
-1.89 \\
\end{tabular} & $\begin{array}{l}.09 \\
.05 \\
\end{array}$ & $\begin{array}{l}.22 \\
.02\end{array}$ & \begin{tabular}{|l|}
.00 \\
.00
\end{tabular} & $\begin{array}{l}13.28 \\
1.43\end{array}$ \\
\hline Variables & & $B$ & $B$ & $t$ & $p$ & $R^{2}$ & $R_{\text {ener. }}^{\prime 2}$ & $F$ \\
\hline $\begin{array}{l}\text { Stap } 1 . \\
\text { Age } \\
\text { Gender } \\
\text { Marital Status }\end{array}$ & $\begin{array}{l}T \\
D \\
T \\
D \\
T \\
D \\
T \\
D\end{array}$ & $\begin{array}{l}-.005 \\
-.10 \\
.071 \\
-.90 \\
.733 \\
.31 \\
\end{array}$ & $\begin{array}{l}-.006 \\
-.08 \\
.005 \\
-.02 \\
.05 \\
-.01\end{array}$ & $\begin{array}{l}-.09 \\
-1.35 \\
.08 \\
-.44 \\
.77 \\
.19\end{array}$ & $\begin{array}{l}.92 \\
.17 \\
.93 \\
.65 \\
.43 \\
.84\end{array}$ & \begin{tabular}{|l|}
.009 \\
.006
\end{tabular} & \begin{tabular}{|l|}
.09 \\
.006
\end{tabular} & $\begin{array}{l}.74 \\
.68\end{array}$ \\
\hline $\begin{array}{l}\text { Step } 2 . \\
\text { Family-Friendly Organizations } \\
\text { Mastery Experiences }\end{array}$ & $\begin{array}{l}T \\
D \\
T \\
D \\
T \\
D \\
\end{array}$ & $\begin{array}{l}.49 \\
.16 \\
.65 \\
.48 \\
\end{array}$ & $\begin{array}{l}.11 \\
.01 \\
.31 \\
.09 \\
\end{array}$ & $\begin{array}{l}2.21 \\
.32 \\
5.95 \\
1.76 \\
\end{array}$ & $\begin{array}{l}.02 \\
.74 \\
.000 \\
.07 \\
\end{array}$ & \begin{tabular}{|l|}
.12 \\
.01
\end{tabular} & $\begin{array}{l}.12 \\
.01\end{array}$ & $\begin{array}{l}7.63 \\
1.09\end{array}$ \\
\hline $\begin{array}{l}\text { Step } 3 . \\
\text { Fam. Erien. Organ X Mastery Exper. }\end{array}$ & $\begin{array}{l}\mathrm{T} \\
\mathrm{D} \\
\mathrm{T} \\
\mathrm{D}\end{array}$ & $\begin{array}{l}-.02 \\
.06 \\
\end{array}$ & $\begin{array}{l}-.09 \\
.12\end{array}$ & $\begin{array}{l}-.37 \\
.42 \\
\end{array}$ & $\begin{array}{l}.71 \\
.67 \\
\end{array}$ & \begin{tabular}{|l|}
.12 \\
.01
\end{tabular} & $\begin{array}{l}.00 \\
.00\end{array}$ & $\begin{array}{l}6.54 \\
.93\end{array}$ \\
\hline Variables & & $B$ & $\beta$ & $t$ & $p$ & $R^{2}$ & $R_{\text {enen }}^{\prime 2}$ & $F$ \\
\hline $\begin{array}{l}\text { Step } 1 . \\
\text { Age } \\
\text { Gender } \\
\text { Marital Status }\end{array}$ & $\begin{array}{l}T \\
D \\
T \\
D \\
T \\
D \\
T \\
D \\
\end{array}$ & $\begin{array}{l}-.005 \\
-.10 \\
.071 \\
-.90 \\
.733 \\
.31 \\
\end{array}$ & $\begin{array}{l}-.006 \\
-.08 \\
.005 \\
-.02 \\
.05 \\
-.01 \\
\end{array}$ & $\begin{array}{l}-.09 \\
-1.35 \\
.08 \\
-.44 \\
.77 \\
.19 \\
\end{array}$ & $\begin{array}{l}.92 \\
.17 \\
.93 \\
.65 \\
.43 \\
.84 \\
\end{array}$ & \begin{tabular}{|l|}
.009 \\
.006
\end{tabular} & \begin{tabular}{|l|}
.09 \\
.006
\end{tabular} & $\begin{array}{l}.74 \\
.68\end{array}$ \\
\hline $\begin{array}{l}\text { Step } 2 . \\
\text { Cultural Values } \\
\text { Mastery Experiences }\end{array}$ & $\begin{array}{l}T \\
D \\
T \\
D \\
T \\
D\end{array}$ & $\begin{array}{l}.20 \\
.20 \\
.65 \\
.48 \\
\end{array}$ & $\begin{array}{l}.17 \\
.06 \\
.31 \\
.09 \\
\end{array}$ & $\begin{array}{l}3.30 \\
1.17 \\
6.01 \\
1.79 \\
\end{array}$ & $\begin{array}{l}.001 \\
.24 \\
.000 \\
.07 \\
\end{array}$ & $\begin{array}{l}.14 \\
.02\end{array}$ & \begin{tabular}{|l|}
.14 \\
.02
\end{tabular} & $\begin{array}{l}8.76 \\
1.35\end{array}$ \\
\hline $\begin{array}{l}\text { Step } 3 . \\
\text { Cult. ValuXMastery Exper. }\end{array}$ & $\begin{array}{l}\mathrm{T} \\
\mathrm{D} \\
\mathrm{T} \\
\mathrm{D}\end{array}$ & $\begin{array}{l}-.03 \\
-.06\end{array}$ & $\begin{array}{l}-.49 \\
-.46 \\
\end{array}$ & $\begin{array}{l}-1.82 \\
-.99\end{array}$ & $\begin{array}{l}.06 \\
.32 \\
\end{array}$ & $\begin{array}{l}.15 \\
.02\end{array}$ & \begin{tabular}{|l|}
.01 \\
.00
\end{tabular} & $\begin{array}{l}8.04 \\
1.29\end{array}$ \\
\hline
\end{tabular}

${ }^{*} \mathrm{~T}=$ Turkish, D=Dutch; ${ }^{*} p<05 ; * *_{p}<01 ; * * 0<<000$ 
Table 6 Moderated Regression Analysis Results for Psychological Well-Being (Social Support, Family-Friendly Organizations, Cultural Values and Control)

\begin{tabular}{|c|c|c|c|c|c|c|c|c|}
\hline Variables & & 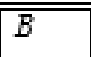 & $\overline{\bar{\beta}}$ & 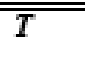 & $\overline{P P}$ & 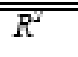 & $\overline{R_{C H A N}}$ & $\overline{F F}$ \\
\hline $\begin{array}{l}\text { Step } 1 . \\
\text { Age } \\
\text { Gender } \\
\text { Marital Status }\end{array}$ & $\begin{array}{l}T \\
D \\
T \\
D \\
T \\
D \\
T \\
D\end{array}$ & $\begin{array}{l}-.005 \\
-.10 \\
.071 \\
-.90 \\
.733 \\
.31 \\
\end{array}$ & $\begin{array}{l}-.006 \\
-.08 \\
.005 \\
-.02 \\
.05 \\
-.01 \\
\end{array}$ & $\begin{array}{l}-.09 \\
-1.35 \\
.08 \\
-.44 \\
.77 \\
.19 \\
\end{array}$ & $\begin{array}{l}.92 \\
.17 \\
.93 \\
.65 \\
.43 \\
.84 \\
\end{array}$ & $\begin{array}{l}.009 \\
.006\end{array}$ & $\begin{array}{l}.009 \\
.006\end{array}$ & $\begin{array}{l}.74 \\
.68\end{array}$ \\
\hline $\begin{array}{l}\text { Step } 2 . \\
\text { Social Support } \\
\text { Control }\end{array}$ & \begin{tabular}{l|}
$T$ \\
$D$ \\
$T$ \\
$D$ \\
$T$ \\
$D$
\end{tabular} & $\begin{array}{l}.25 \\
.11 \\
.53 \\
.62 \\
\end{array}$ & $\begin{array}{l}.32 \\
.09 \\
.25 \\
.11\end{array}$ & $\begin{array}{l}6.22 \\
1.76 \\
4.83 \\
2.06 \\
\end{array}$ & $\begin{array}{l}.000 \\
.07 \\
.000 \\
.03 \\
\end{array}$ & \begin{tabular}{|l|}
.21 \\
.03
\end{tabular} & $\begin{array}{l}.21 \\
.03\end{array}$ & $\begin{array}{l}14.03 \\
2.19\end{array}$ \\
\hline $\begin{array}{l}\text { Step } 3 . \\
\text { Soci Sup.X Control }\end{array}$ & \begin{tabular}{l|}
$\mathrm{T}$ \\
$\mathrm{D}$ \\
$\mathrm{T}$ \\
$\mathrm{D}$ \\
\end{tabular} & $\begin{array}{l}.01 \\
-.006 \\
\end{array}$ & $\begin{array}{l}.44 \\
-.10 \\
\end{array}$ & $\begin{array}{l}1.28 \\
-.25 \\
\end{array}$ & \begin{tabular}{|l}
.20 \\
.80 \\
\end{tabular} & \begin{tabular}{|l|}
.21 \\
.03
\end{tabular} & $\begin{array}{l}.00 \\
.00\end{array}$ & $\begin{array}{l}12.29 \\
1.83\end{array}$ \\
\hline \begin{tabular}{|l|} 
Variables \\
\end{tabular} & & $B$ & $\beta$ & $t$ & $p$ & $R^{2}$ & $R_{\text {ener. }}^{2}$ & $F$ \\
\hline $\begin{array}{l}\text { Step } 1 . \\
\text { Age } \\
\text { Gender } \\
\text { Marital Status }\end{array}$ & $\begin{array}{l}T \\
D \\
T \\
D \\
T \\
D \\
T \\
D\end{array}$ & $\begin{array}{l}-.005 \\
-.10 \\
.071 \\
-.90 \\
.733 \\
.31 \\
\end{array}$ & $\begin{array}{l}-.006 \\
-.08 \\
.005 \\
-.02 \\
.05 \\
-.01 \\
\end{array}$ & $\begin{array}{l}-.09 \\
-1.35 \\
.08 \\
-.44 \\
.77 \\
.19 \\
\end{array}$ & $\begin{array}{l}.92 \\
.17 \\
.93 \\
.65 \\
.43 \\
.84 \\
\end{array}$ & \begin{tabular}{|l|}
.009 \\
.006
\end{tabular} & $\begin{array}{l}.09 \\
.006\end{array}$ & $\begin{array}{l}.74 \\
.68\end{array}$ \\
\hline $\begin{array}{l}\text { Step } 2 . \\
\text { Family-Friendly Organizations } \\
\text { Control }\end{array}$ & $\begin{array}{l}T \\
D \\
T \\
D \\
T \\
D \\
\end{array}$ & $\begin{array}{l}.42 \\
.08 \\
.66 \\
.73 \\
\end{array}$ & $\begin{array}{l}.10 \\
.01 \\
.31 \\
.13 \\
\end{array}$ & $\begin{array}{l}1.89 \\
.16 \\
5.86 \\
2.32 \\
\end{array}$ & $\begin{array}{l}.05 \\
.86 \\
.000 \\
.02 \\
\end{array}$ & .12 & $\begin{array}{l}.12 \\
.02\end{array}$ & $\begin{array}{l}7.45 \\
1.55\end{array}$ \\
\hline $\begin{array}{l}\text { Step } 3 . \\
\text { Fam Frien. Organ X Control }\end{array}$ & \begin{tabular}{l|}
$\mathrm{T}$ \\
$\mathrm{D}$ \\
$\mathrm{T}$ \\
$\mathrm{D}$ \\
\end{tabular} & $\begin{array}{l}.10 \\
.19 \\
\end{array}$ & $\begin{array}{r}.39 \\
.36 \\
\end{array}$ & $\begin{array}{l}1.39 \\
1.14 \\
\end{array}$ & \begin{tabular}{|l}
.16 \\
.25 \\
\end{tabular} & $\begin{array}{l}.13 \\
.02\end{array}$ & $\begin{array}{l}.01 \\
.00\end{array}$ & $\begin{array}{l}6.68 \\
1.51\end{array}$ \\
\hline Variables & & $B$ & $\beta$ & $t$ & $p$ & $R^{2}$ & $R_{\text {ener. }}^{\prime}$ & $F$ \\
\hline $\begin{array}{l}\text { Step } 1 . \\
\text { Age } \\
\text { Gender } \\
\text { Marital Status }\end{array}$ & $\begin{array}{l}T \\
D \\
T \\
D \\
T \\
D \\
T \\
D \\
\end{array}$ & $\begin{array}{l}-.005 \\
-.10 \\
.071 \\
-.90 \\
.733 \\
.31 \\
\end{array}$ & $\begin{array}{l}-.006 \\
-.08 \\
.005 \\
-.02 \\
.05 \\
-.01 \\
\end{array}$ & $\begin{array}{l}-.09 \\
-1.35 \\
.08 \\
-.44 \\
.77 \\
.19 \\
\end{array}$ & $\begin{array}{l}.92 \\
.17 \\
.93 \\
.65 \\
.43 \\
.84 \\
\end{array}$ & $\begin{array}{l}.009 \\
.006\end{array}$ & $\begin{array}{l}.09 \\
.006\end{array}$ & $\begin{array}{l}.74 \\
.68\end{array}$ \\
\hline $\begin{array}{l}\text { Step } 2 . \\
\text { Cultural Values } \\
\text { Control }\end{array}$ & \begin{tabular}{l|}
$T$ \\
$D$ \\
$T$ \\
$D$ \\
$T$ \\
$D$ \\
\end{tabular} & $\begin{array}{l}.17 \\
.17 \\
.64 \\
.68 \\
\end{array}$ & $\begin{array}{l}.14 \\
.05 \\
.30 \\
.12 \\
\end{array}$ & $\begin{array}{l}2.79 \\
1.00 \\
5.76 \\
2.28 \\
\end{array}$ & \begin{tabular}{|l}
.006 \\
.31 \\
.000 \\
.02 \\
\end{tabular} & \begin{tabular}{|l|}
.13 \\
.02
\end{tabular} & $\begin{array}{l}.13 \\
.02\end{array}$ & $\begin{array}{l}8.24 \\
1.75\end{array}$ \\
\hline $\begin{array}{l}\text { Step } 3 . \\
\text { Cult. ValuX X Control }\end{array}$ & \begin{tabular}{l|}
$\mathrm{T}$ \\
$\mathrm{D}$ \\
$\mathrm{T}$ \\
$\mathrm{D}$ \\
\end{tabular} & $\begin{array}{l}-.005 \\
.03 \\
\end{array}$ & $\begin{array}{l}-.09 \\
.23\end{array}$ & $\begin{array}{l}-.30 \\
.53\end{array}$ & \begin{tabular}{|l}
.75 \\
.59 \\
\end{tabular} & \begin{tabular}{|l|}
.13 \\
.02
\end{tabular} & $\begin{array}{l}.00 \\
.00\end{array}$ & $\begin{array}{l}7.06 \\
1.51\end{array}$ \\
\hline
\end{tabular}

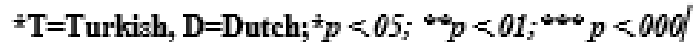




\section{Discussion}

The purpose of the study was to compare recovery and psychological well-being level and examined the role of recovery strategies in moderating the relationship between in social support, family-friendly organizations, cultural values and psychological well-being among the Turkish and Dutch nurses. Additionally to examine whether the moderating roles of recovery strategies will differentiate into a relationship between social support, family-friendly organizations, cultural values and psychological well-being among the both samples. Turkish and Dutch nurses were not comparable with respect to the level of recovery strategies and psychological well-being.

This study showed that social support predicts psychological well-being in our sample. We assume that having a social support (from family, friend etc.) is a positive experience regardless of cultural differences and that it promotes psychological well-being in both Dutch and Turkish nurses. We find interesting differences between Turkish and Dutch nurses. Research results showed that social support had an interaction effect with psychological detachment and relaxation on psychological well-being in the Turkish nurses, whereas in the Dutch nurses only social support and mastery experiences interacted on psychological well-being.

Contrary to our expectations, we did not find a significant interaction between family-friendly organizations, cultural values and recovery strategies on psychological well-being. This might be due to the fact that there was direct relationship between independent variables and the outcome variable of the study without role of recovery.

In general, the results partially have shown that dimensions of recovery strategies have moderator role between social support, family-friendly organizations, cultural values and psychological well-being. In line with our expectations, Turkish nurses report lower levels of recovery, and psychological well-being than the Dutch nurses. However, existing cultural differences between eastern and the western countries in well-being (Liu, Chiu \& Chang, 2017) may also contribute to the cross-national differences found. Cross-cultural studies of well-being consistently find notable differences between collectivistic and individualistic cultures. People who are living in European countries (eg. The Netherlands) report higher satisfaction with life compared with the people who are living in Asian countries (Turkey). This powerful finding has been replicated using a variety of well-being measures in different populations (Cheng et al., 2011; Kim, Schimmack, \& Oishi, 2012).

In line with our expectations, Dutch nurses use recovery strategies more effectively and have more life satisfaction than Turkish nurses. This result might be attributed to differences in subjective factors, such as perceptions of working as a nurse in the society. For example, nursing is considered traditionally in Turkey as a low-status assistant healthcare employee, whilst in the Netherlands it is recognized as an independent profession.

The study does however have several limitations. First, we used a cross-sectional study design - therefore, we may not draw any conclusions about causality. For example, correlations between study variables might be attributable to third variables; thus, with cross-sectional design we cannot address all causality issues satisfactorily. Here, longitudinal or experimental studies are required. ,Second, we conducted our study with participants from a single specific profession which may limit the ability to generalize our findings. Nursing is characterizedby a high degree workload and high timepressure which might make the achievement of psychological well-being particularly difficult. Consequently, further research should study other professions and should examine whether findings generalize to different work settings.

On the basis of our findings, it is advisable to focus interventions on enhancing using recovery strategies and wellbeing. Specific interventions should be directed at training in relaxation techniques detachment from the work in free time. The findings, for both countries, reinforce one of our conclusions that to have a social support, is an important facilitator for psychological well-being among employees. This has an important implication for managers of the organizations/hospitals to create supportive work settings for nurses and all other employees.

\section{References}

Aiken, L. S., West, S. G., \& Reno, R. R. (1991). Multiple regression: Testing and interpreting interactions. Sage.

Bakker, A. B., Demerouti, E., \& Euwema, M. C. (2005). Job resources buffer the impact of job demands on burnout. Journal of Occupational Health Psychology, 10, 170-180.

Browne, M. W., \& Cudeck, R. (1993). Alternative ways of assessing model fit. Sage focus editions, 154, 136-136.

Cheng, C., Jose, P. E., Sheldon, K. M., Singelis, T. M., Cheung, M. W. L., Tiliouine, H., \& Sims, C. (2011). Sociocultural differences in self-construal and subjective well-being: A test of four cultural models. Journal of Cross-Cultural Psychology, 42, 832-855.

Demerouti, E., Bakker, A. B., Geurts, S. A. E., \& Taris, T. W. (2009). Daily recovery from workrelatedeffort during nonwork time. In S. Sonnentag, P. Perrewe, \& D. Ganster (Eds.), Research in occupational stress and well-being: Current perspectives on job-stress recovery (vol. 7,pp. 85-123). Bingley: JAI Press. 
Diener, E. (2000). Subjective well-being. The science of happiness and a proposal for a national index. American Psychologist, 55, 34-43.

Diener, E., Wirtz, D., Tov, W., Kim-Prieto, C., Choi, D. W., Oishi, S., \& Biswas-Diener, R. (2010). New well-being measures: Short scales to assess flourishing and positive and negative feelings. Social Indicators Research, 97(2), 143-156.

European Observatory on Health Care Systems, 2011. Health Care Systems in Transition (Turkey).

Gelfand, M. J., Raver, J. L., Nishii, L., Leslie, L. M., Lun, J., Lim, B. C. et al, (2011). Differences Between

Tight and Loose Cultures: A 33-Nation Study. Science,332, 1100.

Greenberger, E., Goldberg, W. A., Hamill, S., O’Neil, R., \& Payne, C. K. (1989). Contributions of a supportive work environment to parents' well-being and orientation to work. American Journal of Community Psychology, 17, 755783.

Hofstede, G. (1980). Culture's Consequences. Sage, Beverly Hills, CA.

House, R. J., Hanges, P. J., Ruiz-Quintanilla, S. A., Dorfman, P. W., Javidan, M., Dickson, M. W., Gupta,

V., Kennedy, J. C., et al (1999). Cultural Influences on Leadership and Organizations: Project GLOBE. In W. H. Mobley, M. J. Gessner, \& V. Arnold (Eds.), Advances In Global Leadership: 171-233. Stamford, CN: JAI Press.

Kim, H., Schimmack, U., \& Oishi, S. (2012). Cultural differences in self- and otherevaluations and well-being: A study of European and Asian Canadians. Journal of Personality and Social Psychology, 102, 856-873.

Kitayama, S., \& Markus, H. R. (2000). The pursuit of happiness and the realization ofsympathy: Cultural patterns of self, social relations, and well-being. In E. Diener \& Suh, E. (ed.). Subjective well-being across Cultures. Cambridge, MA: MIT Press.

Kossek, E. E., Valcour, M., \& Lirio, P. (2013). The sustainable work force. Organizational strategies for promoting work-life balance and wellbeing. John Willey \& Sons, Ltd. Publishedpp. 295-319.

Liu, Cheng-Hong, Yi-Hsing Claire Chiu, \& Jen-Ho Chang. (2017). "Why do Easterners have lower well-being than Westerners? The role of others' approval contingencies of self-worth in the cross-cultural differences in subjective well-being." Journal of Cross-Cultural Psychology 48(2), 217-224.

Lu, L., Gilmour, R., and Kao, S. (2001). Cultural values and happiness: An east-west dialogue. The Journal of Social Psychology, 141, 477-493.

Marsh, H. W., Hau, K. T., \& Wen, Z. (2004). In search of golden rules: Comment on hypothesis-testing approaches to setting cut-off values for fit indexes and dangers in overgeneralizing Hu and Bentler's (1999) findings. Structural equation modeling, 11(3), 320-341.

Schaufeli, W. B., \& Janczur, B. (1994). Burnout among nurses: a Polish-Dutch comparison. Journal of Cross-Cultural Psychology 25, 95-113.

Siu, O. L., Cooper, K. L., \& Phillips, D. R. (2014). Intervention studies on enhancing work well-being, reducing burnout, and improving recovery experiences among Hong Kong health care workers and teachers. International Journal of Stress Management, 21, 69-84.

Sonnentag, S., \& Fritz, C. (2007). The recoveryExperience questionnaire: Development and validation of ameasure for assessing recuperation and unwinding from work. Journal of Occupational Health Psychology, 12(3), 204-221.

Sonnentag, S., \& Zijlstra, F. R. H. (2006). Job characteristics and off-job time activities as predictors forneed for recovery, well-being and fatigue. Journal of Applied Psychology, 91, 123-142.

Thompson, C. A., \& Prottas, D. J. (2005). Relationships among organizational family support, jobautonomy, perceived control, and employee well-being. Journal of Occupational Health Psychology, 10 (4), 100-118.

Walsh, J. K., \& Lindblom, S. S. (2000). Psychophysiology of sleep deprivation and disruption. In M. R.

Pressman \& W. C. Orr (Eds.)Understanding sleep: The evaluation and treatment of sleep disorders(pp. 73 - 110). Washington, DC: APA.

Zijlstra, F. R. H. (1993).Efficiency in work behaviour: A design approach for modern tools $(\mathrm{PhD}$ thesis, Delft University of Technology). Delft, The Netherlands: Delft University Press.

Zijlstra, F. R. H., Cropley, M., \& Rydstedt, L.W. (2014). From recovery to regulation: An attempt to

Reconceptualize 'recovery from work'. Stress and Health, 30, 244-252.

Zijlstra, F.R.H., \& Sonnentag, S. (2006). After work is done: Psychological perspective on recovery fromwork. European Journal of Work and Organizational Psychology, 15, 129-138.

Zimet, G. D., Dahlem, N.W., Zimet, S.G., \& Farley, G.K. (1988). The multidimensional scale of perceived social support. Journal of Personality Assessment. 52, 30-41. 